\title{
Multiple gastrointestinal complications in Marfan syndrome
}

\author{
Ron Eliashar, Jean-Yves Sichel, Adi Biron, Itzhak Dano
}

\begin{abstract}
A 36-year-old woman with Marfan syndrome came to the Ear, Nose and Throat clinic complaining of severe dysphagia and regurgitation of ingested food from which she had been suffering for the past three years. She had undergone Nissen's fundoplication for hiatal hernia repair at the age of 20 years, a sigmoidectomy for colonic diverticulosis with perforation of a diverticulum when she was 25 years old, and small bowel resection due to small bowel obstruction 3 years later. The patient's history also included inguinal hernia repair, mitral valve prolapse, mild congestive heart failure, atrial fibrillation, and hypothyroidism. Upon examination, an obvious Marfanoid habitus was noted with severe kyphoscoliosis and pectus excavatum, and a 3/6 systolic murmur at the apex was detected. Her abdomen was marked with multiple scars. Chest X-ray showed severe kyphoscoliosis. A computed tomography (CT) scan of the neck and chest was performed (figure). The patient was then operated on under general anaesthesia and a diverticulectomy and cricopharyngeal myotomy were performed. Postoperatively, she managed well with improvement of the dysphagia and no regurgitation of ingested food.
\end{abstract}

\section{Department of Otolaryngology/Head \\ \& Neck Surgery, \\ Hadassah University Hospital, Jerusalem, Israel \\ R Eliashar \\ J-Y Sichel \\ A Biron \\ I Dano}

Accepted 10 March 1998

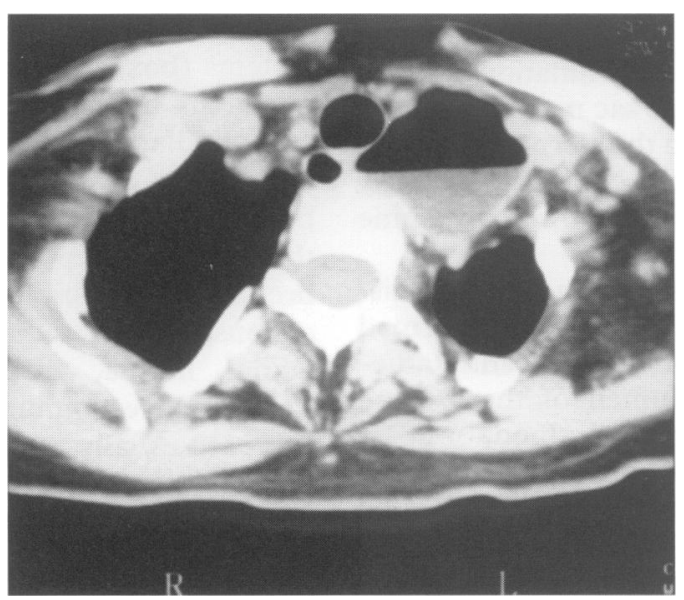

\section{Questions}

1 What is the most likely diagnosis on the basis of the CT appearance?

2 List at least five symptoms of Marfan syndrome.
Figure A CT-scan of the chest showing an air-fluid level in a sac extending into the left side of the chest 
Answers

QUESTION 1

A CT-scan of the neck and chest with contrast material shows a sac containing an air-fluid level and extending into the left side of the chest (figure). This is compatible with a Zenker's diverticulum. The upper oesophageal sphincter is composed of the cricopharyngeal muscle, which is in fact the lower horizontal part of the inferior constrictor muscle. ${ }^{1}$ Above the upper oesophageal sphincter is an area which is relatively weak and unable to sustain too much pressure. It is known as Killian's Dehiscence. This is the site of herniation of a Zenker's diverticulum, which is actually a pseudodiverticulum, since only the mucosa and the submucosa herniate (unlike a true diverticulum which consists of all layers of the wall). ${ }^{3}$ It is a pulsion diverticulum, because it is associated with high intraluminal pressure, ${ }^{13}$ and it is usually found on the left hand side. ${ }^{12}$ Usually it appears during the sixth through the ninth decade of life, with the following symptoms: long-standing dysphagia of insidious onset, spontaneous regurgitation of undigested food, foul breath, aspiration and coughing, hoarseness, and obstruction of the oesophageal lumen when the sac is enlarged.

Generally, the physical examination is unremarkable, although a large sac may appear as a cervical mass posterior to the sternocleidomastoid muscle, and neck compression may produce a gurgling noise followed by some coughing. ${ }^{13}$

Diagnosis on the basis of the typical history, is generally easy and can be confirmed by a barium swallow or a CT scan. Radiographic defects in the lumen of the sac are usually caused by retained food, but if they remain unchanged after repeated examinations a carcinoma should be considered. ${ }^{134}$

Management depends on the rate of advancement of the disease: small asymptomatic diverticula require no treatment, while symptomatic diverticula require surgery. External approaches include cricopharyngeal myotomy alone, diverticulectomy with or without cricopharyngeal myotomy, and diverticulopexy with or without cricopharyngeal myotomy. ${ }^{13}$ Even large sacs and protruding retrosternal sacs can be managed through the neck by using one's finger, as was done in our case. Sometimes, a sternotomy is necessary. Endoscopic surgery includes removal of the common oesophageal lumen-diverticulum wall using diathermy or laser (Dohlman's procedure) and dilatation. ${ }^{13}$

QUESTION 2

The most common symptoms of Marfan syndrome are shown in the box. Marfan syndrome is a connective tissue disorder which is usually manifested in the skeletal, ocular and cardiovascular systems. Gastrointestinal tract abnormalities are uncommon and only a few cases have previously been reported.
Common manifestations of Marfan syndrome

- skeletal: elongated extremities, kyphoscoliosis, pectus excavatum or curvinatum, loose jointedness

- ocular: ectopia lentis, retinal detachment

- aorta: dilatation, aneurysm, aortic regurgitation

\section{Discussion}

Marfan syndrome is a connective tissue disorder inherited as an autosomal dominant trait with almost complete penetration. ${ }^{5-7}$ Recently, the Marfan syndrome locus was traced to chromosome 15 (q15-q21). ${ }^{7}$ Almost simultaneously, Hollister et al, ${ }^{8}$ reported that patients with Marfan syndrome lacked fibrillin, a component of the microfibrillar system associated with elastin, in their skin. Lee $e t a l^{9}$ confirmed the hypothesis that these two observations are linked. Thus, according to the current concept, Marfan syndrome is caused by mutations in the fibrillin gene. ${ }^{59}$

The disorder manifests in multiple organ systems (box), but gastrointestinal tract complications are quite uncommon. Only a few cases of diaphragmatic or inguinal hernia, and one case of diverticulosis coli have previously been reported. ${ }^{10}$ To the best of our knowledge, this is the first report on a case of Marfan syndrome with multiple gastrointestinal complications, namely, diaphragmatic hernia, inguinal hernia, diverticulosis coli, and a Zenker's diverticulum. The last two complications are usually associated with older people. Their appearance in a comparatively young person, along with the associated Marfan syndrome, suggests the possibility of some linkage between them. Unfortunately, we have no pathological specimen of the colonic diverticula, and since the Zenker's diverticulum is a pseudodiverticulum, we do not have any information on the histologic structure of the gastrointestinal wall. Suster et al, who reported the diverticulosis coli, ${ }^{10}$ noted a thinning of the wall with an absence of the muscularis propria and non-specific inflammation. We can only speculate the existence of an abnormally weak wall in the entire gastrointestinal tract, which may be related to the connective tissue disorder. We therefore recommend a special awareness of gastrointestinal tract complications in the treatment of patients with Marfan syndrome.

\section{Final diagnosis}

Zenker's diverticulum and other gastrointestinal complications in a patient suffering from Marfan syndrome.

Keywords: Marfan syndrome; Zenker's diverticulum; diverticulosis. 
1 Doyle PJ, Stevens HE. Esophageal diverticula. In: Cummings $\mathrm{CW}$, ed, Otolaryngology-head and neck surgery. S Luois: Mosby-Year Book Inc, 1993; pp 2369-81.

2 Ertekin C, Pehlivan M, Aydogou I, et al. An electrophysiological investigation of deglutition in man. Muscle Nerve 1995;18:1177-86.

3 Shockley WW. Esophageal disorders. In: Bailey BJ, ed, Head and neck surgery - otolaryngology. Philadelphia: JB Lippincott, 1993; pp 690-710.

4 Nilsson ME, Isberg A, Schiratzki $H$. The hypopharyngeal diverticulum. A simultaneous cineradiographic and manometric examination. Acta Otolaryngol Stockh 1988;106:314 20 .

5 Tsipouras P. Marfan syndrome: a mystery solved. $\mathcal{f}$ Med Genet 1992;29:73-4.
6 Judo KP. Hyperelasticity syndromes. Cutis 1984;33:494-6. 7 Kainulainen K, Pulkkinen L, Savolainen A, Kaitila I, Peltonen L. Location on chromosome 15 of the gene defect causing Marfan's syndrome. N Engl F Med 1990;323 935-9.

8 Hollister DW, Godfrey M, Sakai LY, Pyreritz RE. Immunohistologic abnormalities of the microfibrillar-fiber system in histologic abnormalities of the microfibrillar-fiber system
the Marfan syndrome. N Engl $\mathcal{F}$ Med 1990;323:152-9.

9 Lee B, Godfrey M, Vitale E, et al. Linkage of Marfan's syndrome and a phenotypically related disorder to two differen fibrillin genes. Nature 1991;352:330-4.

10 Suster SM, Ronnen M, Bubis JJ. Diverticulosis coli in association with Marfan's syndrome. Arch Intern Med 1984; 144:203.

\title{
Recurrent infections and multiple bone fractures in a 4-year-old child
}

\author{
Ashok Vaghjimal, Jenny Shliozberg, Arye Rubinstein
}

A 4-year-old boy was referred to our clinic for evaluation of repeated skin infections, pneumonias, and recurrent otitis media starting from 9 months of age. The child had a history of eczema from one week of age. At the age of 3.5 years, he had sustained three fractures of long bones after a minor trauma. The family history was unremarkable. On physical examination he had an unusual and typical facies (figure), onychomycosis of finger nails and fading eczema on the right forearm. The rest of the physical examination was normal. The white cell count was elevated at $20.0 \times 10^{9} / 1$ with $20 \%$ eosinophils. Serum IgG, A and $M$ were normal. T and B cells were also within normal limits, both as percentages and in absolute numbers. Delayed-type hypersensitivity skin tests with Candida, tetanus toxoid and purified protein derivative were negative.

\section{Albert Einstein \\ College of Medicine, \\ Division of Allergy and \\ Immunology, Mazer \\ Building Room 200, \\ 1300 Morris Park \\ Avenue, Bronx, NY \\ 10461, USA \\ A Vaghjimal \\ J Shliozberg \\ A Rubinstein}

Accepted 10 March 1998

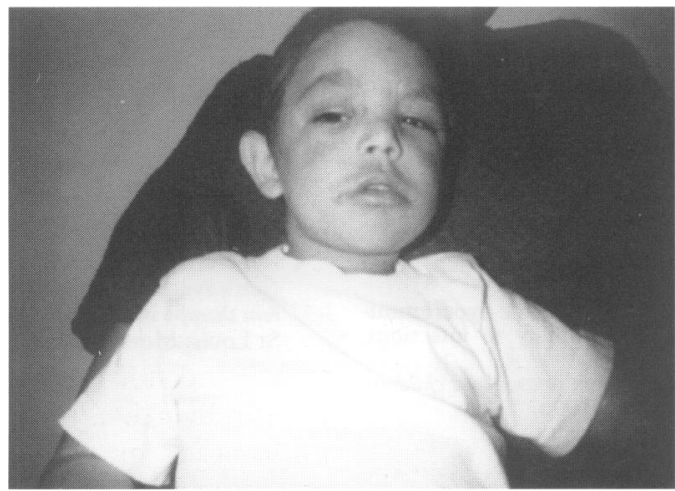

\section{Questions}

1 What tests should be performed?

2 What is the probable diagnosis? 